\title{
Careers in academic medicine
}

\author{
Mark AJ Devonald
}

Mark AJ

Specialist Registrar in Nephrology,

Norfolk and

Norwich University

Hospital

This conference was held at the Royal College of Physicians on 19 February 2004

Clin Med 2004;4:284-5
Devonald MRCP

At this meeting at the Royal College of Physicians (RCP), established academics and members of grant awarding bodies advised an audience, predominantly comprising trainees pursuing or contemplating a career in clinical science, on why, when and how to tackle such a career path in the current climate.

There has arguably never been a more exciting time to be involved in clinical science. In this era of genomics and proteomics, disease-causing genes are being identified, mechanisms of disease elucidated, and targeted therapeutic strategies developed. At the population end of the research spectrum, large scale clinical trials are facilitating the practice of evidencebased medicine.

When discussing reasons to embark on an academic career, this was perhaps preaching to the converted. Clearly there exists a variety of motivating factors between individuals, but common to many, the fascination, occasionally the addiction, of enquiry. To ask the correct question, the first and most critical step, and then to devise methods to answer it.

Equally important, though, are reasons not to undertake research. How many research theses have been written not because of interest in the subject

\section{Conference programme}

I How to plan your career in academic medicine Professor Mark Walport, The Wellcome Trust

\section{Personal Experiences}

Dr Fiona Karet, Wellcome Trust Senior Clinical Fellow, Cambridge Institute for Medical Research.

Dr Duncan Bassett, MRC Clinical Scientist Fellow, Imperial College, London

- The Academy of Medical Sciences

Professor Michael Orme, Chairman, Academic Careers Committee, Academy of Medical Sciences

- Balancing your Clinical and Research Training Professor Chris Pugh, The Wellcome Trust Centre for Human Genetics, University of Oxford

I Research Funding Agencies

Medical Research Council - Dr Robert Bennett

The Wellcome Trust - Dr John Williams

Cancer Research UK - Professor Robert Souhami

British Heart Foundation - Professor Sir Charles George

National Co-ordinating Centre for Research Capacity Development Dr Lisa Cotterill itself, but as a perceived means to reach the next step of a clinical career? This issue is becoming more topical with changes in training schemes, particularly with the trend towards shortening the duration of clinical training. Feeling obliged to undertake research is not only undesirable from the trainee's perspective, taking time away from their preferred activities, but it also removes limited resources from others who do have a genuine interest in developing a research career. There may be a case here for greater availability of one year MSc courses, either to provide subspecialty teaching, or to provide an introduction to research methodology to those who wish to be 'research aware' but do not wish to undertake a thesis. Nevertheless, research degrees continue to serve as hard currency in the clinical jobs market.

Of greater concern are the disincentives to pursuing clinical science for those trainees who do have a genuine interest. The frequently cited evidence for the existence of a problem is the number of senior academic positions in the UK, which remain unfilled. Since competition for externally funded training (doctoral) and intermediate (post-doctoral) fellowships remains intense, the conversion of postdoctoral fellows or university post holders (clinical lecturers) to senior positions appears to be an area of susceptibility. This may be a consequence of both an inadequate number of posts and of a trickle of such post-holders back to NHS positions.

This recruitment and retention problem was noted almost 10 years ago, when the House of Lords Select Committee on Science and Technology expressed concern about the disincentives to an academic medical career. Further complications have since arisen, following significant changes to clinical training with the introduction of the specialist registrar grade. In March 2000 a working party from The Academy of Medical Sciences considered the threats to academic medicine and made seven recommendations, including a key proposal, the introduction of the tenure-track clinician scientist post (the 'Savill Report'). Discussion of these proposals, with some analysis of their effect some four years on, emerged as one of the main themes of this meeting. ${ }^{1}$

The Savill Report expressed concern at the declining numbers of clinical lecturers. It suggested that as a consequence of the research assessment exercise (RAE), whereby an academic department's rating influences its income, there may be pressure on medical schools to replace these training posts 
with non-training posts which might be more research productive in the short term, such as non-clinical scientists or senior lecturers. The Report also identified three general disincentives to training in academic medicine compared with clinical training programmes:

(i) lack of a clear career structure,

(ii) inflexibility in combining clinical and research training

(iii) the prolonged insecurity of clinical academic training.

Further issues were noted in certain disciplines. For example in the 'craft' specialties of surgery, obstetrics and gynaecology, and in some medical specialties, prolonged periods of research, if not integrated with clinical training, could result in diminution of practical skills.

The Report recommended maintaining two stages of research training, namely a doctoral phase, preferably funded by winning a research training fellowship, and a post-doctoral phase. The latter is now generally recognised as a prerequisite to taking up a senior post as an independent investigator in a competitive discipline. It was recommended that the two established means of undertaking post-doctoral research, the clinical lectureship and the intermediate fellowship, should continue, as these may be appropriate for some individuals. However, since neither of these routes was considered to overcome the disincentives listed above, the Report proposed the introduction of the clinician scientist scheme.

It was suggested that this scheme would provide approximately 50 fellowships per year, funded by some redeployment of Higher Education Funding Councils money in addition to input from Medical Research Council and research charities such as the Wellcome Trust. It was envisaged that candidates would have completed a doctorate, and have two or three years' clinical training remaining prior to the award of certificate of completion of specialist training (CCST). The fellowship, generally of five years' duration, would allow the trainee to tailor to their individual requirements, the combination of further research and completion of clinical training. The latter would be in a supernumerary role. It was recommended that, in order to improve career security, tenure-track status should be conferred ie an understanding with the host medical school that a senior academic position should be available on satisfactory completion of the fellowship.

This scheme remains under evaluation, but it is of note, firstly, that after four years, fewer than half of the projected 200 clinician scientists are in post. This appears to reflect a paucity of applications from suitably qualified individuals. Secondly, a proportion of those appointed had already acquired a CCST prior to commencing the scheme, which differs from the stated objectives of the scheme. The outcome of the tenure-track proposal remains to be seen.

So much for politics and the restructuring of the training grades, what advice was offered to this meeting's audience with respect to the timing and planning of research? Firstly it was considered preferable to have had some early exposure to research, for example by undertaking an intercalated BSc. Timing of the doctoral phase can be highly variable, ranging from pre-qualification, as part of an $\mathrm{MB}, \mathrm{PhD}$ programme offered by a few medical schools, to late, a year or two away from award of CCST. There emerged a surprisingly clear consensus from the speakers that most trainees would benefit from undertaking the doctoral phase relatively late, having established themselves, in the case of hospital-based trainees, in a specialist registrar (or clinical lecturer) post with a national training number. No doubt, enthusiastic proponents of the $\mathrm{MB}, \mathrm{PhD}$ programmes do exist, but perhaps were not represented at the meeting. The main disadvantages of an early doctoral phase were considered to be, firstly, that the trainee would probably not have chosen their clinical specialty, which might later render the research project less appropriate. Secondly, a relatively long period of clinical training time would intervene prior to the post-doctoral phase, during which time the relevance of the doctoral research might diminish. Moreover, the trainee might appear less competitive when applying for intermediate or clinician scientist fellowships.

Several clear points of advice also emerged with respect to the general approach to a research project. Whether considering basic research, experimental medicine, or population medicine, the research should be fundamentally driven by the question, rather than by some other factor such as the availability of a particular technique (or as one speaker put it, 'have assay, will travel!'). It therefore follows that not only should the question be an important one, but that it should be answerable - and be seen as such by grants committees.

In most cases, at both doctoral and post-doctoral level, choice of the question will be determined or at least heavily influenced by the supervisor. Choice of supervisor is therefore critical, as in addition to their input into the research project, he or she should be able to provide objective, dispassionate career advice. Ideally, the supervisor should be available, well-funded, productive, and on the way up rather than down. If not clinically qualified, the supervisor should be familiar with clinicians and with the issues of combined scientific and clinical training. They should be able to supervise the doctoral trainee in the acquisition of generic research skills, in learning to ask the right questions rather than simply how to perform the experiments. They should help the post-doctoral trainee to find his or her own niche, in which they can develop expertise and independence. It was emphasised by several speakers, however, that the most objective career advice sometimes comes from a carefully chosen mentor who is independent of the supervisor.

Combining research and clinical medicine will inevitably be more complex and will take longer than clinical medicine alone, if training in both components is to be adequate. Some of the disincentives created by this complexity and duration are being addressed. The message, albeit from those who have succeeded in this competitive field, is that the effort is worth it.

\section{References}

1 The Academy of Medical Sciences. The tenure-track clinician scientist: a new career pathway to promote recruitment into clinical academic medicine, 2000: published on the Academy's web site www.acmedsci.ac.uk 